\title{
The Neurological and Neurosurgical Presentations of SARS- CoV2 Affected Patients: A Systematic Review.
}

\author{
Selina $\mathrm{F}^{1}$, Talha $\mathrm{KA}^{2}$, Ahmed $\mathrm{MT}^{3}$, Karim $\mathrm{IJ}^{4}$, Nasir $\mathrm{M}^{5}$, Latif $\mathrm{S}^{6}$, Perveen $\mathrm{RA}^{7}$, Bhattacharya $\mathrm{R}^{8}$
}

Conflict of interest: There is no conflict of interest relevant to this paper to disclose.

Funding Agency : was not funded by any institute or any group.

Contribution of Authors :

Principal Investigator- Dr. Farhana Selina

Manuscript preparation- Dr. Khandaker Abu Talha, Dr Mostafa Taufiq Ahmed, Dr Ishrat Jahan Karim

Data collection- Prof. Dr. Morshed Nasir, Dr. Sonia Latif.

Editorial formatting- Dr. Rawshan Ara Perveen, Dr. Ramanuj Bhattacharya

Copyright: @2020bang.BJNS published by BSNS. This article is published under the creative commons CC-BY-NC license. This license permits use distribution (https://creativecommons. orgf/licences/by-nc/4-0/)reproduction in any medium, provided the original work is properly cited and is not used for commercial purposes.

Received: 10/09/2020

Accepted: 11/11/2020

\begin{abstract}
:
Presentation with neurological complaint is not very uncommon. Few patients report with intracerebral hemorrhage which are considered as cases of neurosurgical intervention, on the other hand respiratory depression and encephalitis patients need care in Neurosurgical intensive care unit (Neurosurgical ICU) for treating or measuring intracranial pressure (ICP). This systematic review includes 2683 COVID19 patients of 17 articles who had neurological manifestations. Out of these 17 studies 8 articles were retrospective case series, 3 were prospective case series, 3 were case reports, 2 were cross-sectional and 1 cohort study. Headache was commonest neurological feature presented in 7 studies. Cerebro-vascular problem, loss of smell and impaired conscious level was present in four studies each. Seizure was presenting feature in 3 studies. There were series where patients reported with loss of taste. Other presenting features were dizziness and ataxia. Previous studies supported that Corona virus can cause encephalitis, multiple sclerosis or cerebral hemorrhage. Respiratory failure due to damage of cardiorespiratory center of brainstem by COVID19 virus has not been ruled out.
\end{abstract}

Key words: COVID-19, Corona virus (CoV), Neurological manifestation.

Bang. J Neurosurgery 2021; 10(2): 169-174

all the patients had respective lung ground glass opacities on high resolution computed tomography (HRCT) of chest. The underlying side effects included fever $(98 \%)$, cough $(76 \%)$, dyspnea $(55 \%)$, myalgia or weakness (44\%), sputum creation ( $28 \%)$, cerebral pain $(8 \%)$, hemoptysis $(5 \%)$, and loose motion $(3 \%)$. Just a single patient didn't present fever in the onset of illness. Twelve (29\%) cases advanced to intense

1. Dr. Farhana Selina, MBBS, MD (Anesthesiology), Associate Professor of Anesthesiology, Sylhet Women's Medical College, Bangladesh.

2. Dr. Khandaker Abu Talha, MBBS, MS (Neurosurgery), MPH, Diploma in Clinical Research (Canada), Associate Professor of Neurosurgery, Sylhet Women's Medical College, Bangladesh.

3. Dr Mostafa Taufiq Ahmed. MBBS, MS, Assistant Prof. dept of neurosurgery, Sylhet M A G Osmani Medical College

4. Dr Ishrat Jahan Karim. MBBS, MS, Assistant Professor, Dept of Obstetrics \& Gynaecology. Sylhet M A G Osmani Medical College

5. Prof. Dr. Morshed Nasir. MBBS, M. Phil (Pharmacology), FRSPH (Public Health), Professor \& HOD of Pharmacology \& Therapeutics, Holy Family Red Crescent Medical College, Bangladesh.

6. Dr. Sonia Latif. MBBS, DA, Registrar, Anesthesiology, Sylhet Women's Medical College, Bangladesh.

7. Dr. Rawshan Ara Perveen. MBBS, MPhil, Assistant Prof. Pharmacology, Holy Family Red Crescent Medical College.

8. Dr. Ramanuj Bhattacharya, Medical officer, Anesthesiology, Sylhet Women's Medical College, Bangladesh.

Address of Correspondence: Dr. Farhana Selina, Associate Professor of Anesthesiology Sylhet Women's Medical College, Mirboxtola, Sylhet 3100, Bangladesh. Mobile \& WhatsApp: 01747260039, Email: farhana.selina@gmail.com 
respiratory misery condition (ARDS), 5 (12\%) had intense heart injury, $3(7 \%)$ had intense kidney injury $(\mathrm{AKI})$, and $3(7 \%)$ presented with shock. At the information cutoff date, $28(68 \%)$ patients were released and $6(15 \%)$ had died.

It was detected that brain expresses ACE2 receptors (Figure-1). ${ }^{3}$ Viremia (Figure-1 A) helps to spread the COVID-19 infection all through the body by means of the circulatory system (Figure-1 B). Neurotropism may happen by means of flow as well as an upper nasal course that empowers the COVID-19 to arrive at the cerebrum (Figure-1 C) and tie and connect with the ACE2 receptors (Figure-1 D). COVID-19 attaches with the ACE2 by means of spike protein (Figure-1 D) over glial cells and neurons, which makes them a potential objective of COVID-19. The spread of COVID-19 in the fundamental dissemination or over the cribriform plate of the ethmoid bone during an early or later period of the contamination can prompt cerebral association as has been accounted for in the past for SARS-CoV affected patients. The nearness of the COVID-19 infection in the general flow justifiably empowers it to go into the cerebral course (Figure 1) where the sluggish flow of the blood inside the microcirculation could be one of the variables that may encourage the communication of the COVID-19 infection spike protein with ACE2 communicated in the slim endothelium. Ensuing sprouting of the viral particles from the fine endothelium and harm to the endothelial coating can support viral access to the cerebrum (Figure $1 \mathrm{~B}$ ). It is thought that, some time before the neuronal damage occurs, the endothelial rupture in cerebral vessels inside the cerebral tissue can have fatal results in patients with COVID-19 contaminations. The development of the COVID-19 infection to the mind by means of the cribriform plate near the olfactory bulb can be an extra pathway that could empower the infection to reach and affect the cerebrum. Furthermore, the findings like a decreased smell sensation or hyposmia in a simple beginning period COVID19 patient ought to be examined altogether for CNS association. In the SARS-CoV contaminations that were accounted for previously, post-mortem findings of the patients have demonstrated strong evidence of the nearness of SARS-CoV by electron microscopy, immunohistochemistry, and constant opposite transcription PCR. Patients with intense SARS-CoV showed presence of infection in cerebrospinal fluid (CSF). The function of the bloodbrain barrier in containing the infection and keeping it from accessing the neural tissues yet to be confirmed in patients diagnosed as COVID-19. ${ }^{4}$ Moreover, few patients who had loss of automatic control over breathing during the COVID-19 pandemic suffering intense respiratory failure compelled clinicians to isolate COVID-19 patients into neurologically affected cases and the individuals who are without neurological deficits.

\section{Objective and Methodology:}

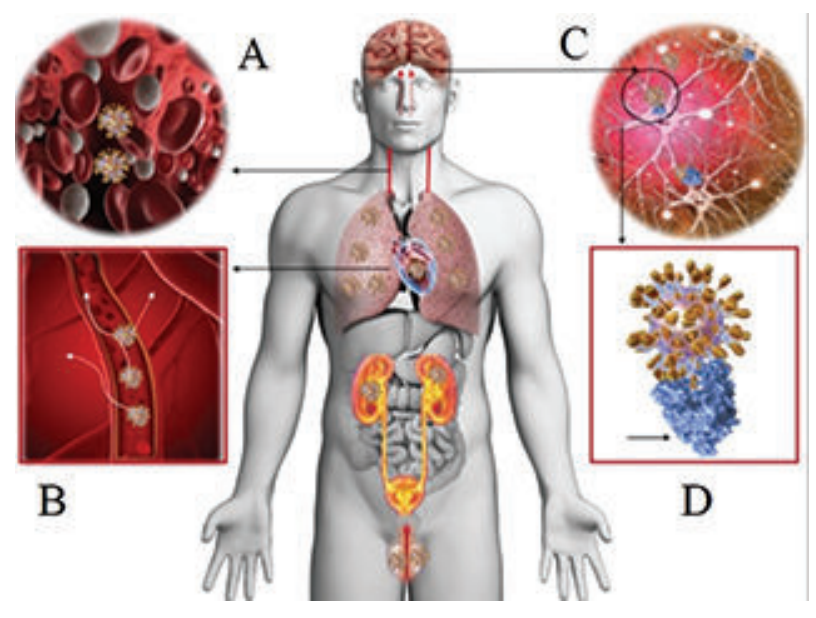

Fig.-1: Showing distribution of ACE2 receptors in different parts of body including brain

Inclusion criteria: Articles on COVID-19 patients suffered from neurological manifestations were included in this study.

Exclusion criteria: Articles on COVID-19 patients with other than neurological manifestations, articles with incomplete information about clinical feature and of low reliability were excluded from the study.

Rationale of the study was confirmed by the researchers after studying related previous studies. Objectives were set according to PICOS guideline. Review question was set as if the COVID-19 patients may develop neurological manifestations or not? All diagnosed cases of covid-19 had neurological manifestations were included in the studies. Protocol was made according to Preferred Reporting Items for Systematic Reviews and Meta-Analyses (PRISMA) guideline. After discussing the methodology, two researchers collected related articles from PubMed, Web of science and Google search engine. Reference manager software Mendeley was used to search from database, sorting according to references and saving and sharing the articles. Individual researchers prepared separate logs using unique IDs. Both the researchers then consulted together and sorted the 
articles according to the references, selection criterion, statistical analysis and outcomes of the articles and moved for the second log. Second log was comprised of references, number of the participants, types of the statistical analysis, outcome measuring and the types of study. Studies accepted by both the researchers were included. Second set of unique IDs were allotted for finally accepted studies. After preparing the final table, all the results of the studies were tabulated and compared.

\section{Results:}

Table -I illustrated that total 17 articles were reviewed. Total 2683 COVID-19 patients were included in these articles. Out of these articles, 8 articles were retrospective case series, 3 were prospective case series, 3 were case reports, 2 were cross-sectional and 1 cohort study. Headache was commonest neurological feature presented in 7 studies. Cerebrovascular problem, loss of smell and impaired conscious level was present in four studies each. Seizure was presenting feature in 3 studies. There were series where patients reported with loss of taste. Other presenting features were dizziness and ataxia.
The review article of Ali AAsadi reviewed the result of 6 articles where all the series had patients with CNS manifestation. ${ }^{5}$ Giacomeli et al found 34 patients reported with loss of smell out of 59 cases. ${ }^{6}$ The sample size of the cohort study of Hopkins et al was 382 and 86 of them had loss of smell. ${ }^{7}$ RCS of Klok et al had enrolled 184 patients and $1.6 \%$ of them reported with cerebro-vascular features. ${ }^{8}$ Lechien et al had 417 patients in their PCS and the presentations of headache, loss of smell and loss of taste were 45,86 and 89 respectively. ${ }^{9}$ Cerebro-vascular presentation was in $2.5 \%$ of the PCS of Lodigiani where total 338 patients were enrolled. ${ }^{10}$ According the study of Mao et al they have done retrospective case series study on 214 admitted patients among whom $25 \%$ had CNS manifestation. Of them $13 \%$ had headache, $17 \%$ had dizziness, $8 \%$ had impair consciousness. Presentations like acute cerebral stroke, ataxia and seizure were in $3 \%, 0.5 \%$ and $0.5 \%$ patients respectively. ${ }^{11}$ In the series of Li et al they performed their retrospective case series on 221 Covid-19 patients. Among the patients $5 \%$ presented with acute ischemic stroke. ${ }^{12}$ Other CNS presentations were

Table-I

Shows different articles on different types neurological manifestations presented by COVID-19 patients.

\begin{tabular}{|c|c|c|c|c|c|c|c|c|c|c|c|}
\hline \multirow[t]{2}{*}{ Author } & \multirow{2}{*}{$\begin{array}{l}\text { Types of } \\
\text { study }\end{array}$} & \multirow[t]{2}{*}{ No. } & \multicolumn{7}{|c|}{ Neurological findings \& percentage } & \multirow[b]{2}{*}{$\begin{array}{c}\text { Taste } \\
\text { lost }\end{array}$} & \multirow[b]{2}{*}{ ANS } \\
\hline & & & Headache & dizziness & $\mathrm{ICL}$ & $\begin{array}{c}\text { CVS } \\
\text { problem }\end{array}$ & Ataxia & Seizure & $\begin{array}{c}\text { Smell } \\
\text { lost }\end{array}$ & & \\
\hline Giacomelli & CS & 59 & & & & & & & 34 & & \\
\hline Hopkins & Cohort & 382 & & & & & & & 86 & & \\
\hline Klok & RCS & 184 & & & & $1.6 \%$ & & & & & \\
\hline Lechien & PCS & 417 & 45 & & & & & & 86 & 89 & \\
\hline Lodigiani & PCS & 338 & & & & $2.5 \%$ & & & & & \\
\hline Mao & RCS & 214 & 13 & 17 & 8 & 3 & 0.5 & 0.5 & & & \\
\hline Lee & RCS & 221 & & & & 6 & & & & & \\
\hline Huang & PCS & 41 & 8 & & & & & & & & \\
\hline Yang & RCS & 52 & 6 & & & & & & & & \\
\hline Wang & RCS & 138 & 7 & 9 & & & & & & & \\
\hline Chen & RCS & 99 & 8 & & 9 & & & & & & \\
\hline Sijia & RCS & 262 & 6.5 & & & & & & & & \\
\hline Yeshun & RCS & 214 & & & & & & & & & 36.5 \\
\hline Sandeep & $\mathrm{CR}$ & 1 & & & & & & 100 & & & \\
\hline Asia & CR & 1 & & & 100 & & & 100 & & & \\
\hline Carol H & CS & 59 & & & & & & & 68 & 71 & \\
\hline $\begin{array}{l}\text { Rong } \\
\text { Total- } 2683\end{array}$ & CR & 1 & & & 100 & & & & & & \\
\hline
\end{tabular}

RCS- retrospective case series, ICL- impaired conscious level, PCS- prospective case series, CR- case report, ANS- average neurological symptoms, CS- cross sectional, 
cerebral venous thrombosis $(0.5 \%)$ and cerebral hemorrhage $(0.5 \%)$. Huang et al published their prospective study on 41 patients of them $8 \%$ were presented with headache. ${ }^{13}$ Yang et al's retrospective study was on 52 critically ill patients and headache was present in $6 \%$ cases. ${ }^{14}$ Retrospective study of Wang et al showed that among 138 patients $9 \%$ had dizziness and $7 \%$ had headache. ${ }^{15}$ In series of Chen et al (total patients 99) they found confusion in 9\% patients and headache in $7 \%$ patients. ${ }^{16} \mathrm{CSF}$ analysis or EEG study was not done in any studies.

\section{Discussion:}

Sijia Tian et al publisher article on the characteristics of Covid-19 patients of Beijing. Among total 262 patients, $17 \%$ were severe cases. ${ }^{17}$ Nearly half of the patients were male and the average age of the patients was 47 years. Commonest presentation at the onset of covid-19 was fever (82.1\%). Other features were cough (45.8\%), headache (6.5\%), dyspnea (6.9) and fatigue $(26.3 \%)$. Sandeep et al reported a case of COVID-19 patient who had developed seizure. ${ }^{18} \mathrm{~A} 72-$ year old man presented with weakness and dizziness who had history of diabetes, chronic kidney disease, hypertension and coronary artery disease. He was reported positive for COVID-19. CT head showed chronic microvascular ischemic changes without any hemorrhage or infarction. CT scan chest revealed right lower lobe consolidation with both sided lung opacity. On day 3 of admission, patient developed multiple episodes of generalized tonic colonic seizure which lasted for few minutes. He was administered levetiracetam, initially loading dose and followed by maintenance dose. A 24-hour EEG revealed left temporal seizures and left temporal sharp waves which were epileptogenic. On the fifth day of hospital admission he became pulseless and collapsed. The resuscitation attempt was failed and the patient died.

According to the case report of Asia et al one 74year-old male was presented with cough and fever. ${ }^{19}$ He was suffering from Parkinson disease, atrial fibrillation, and chronic obstructive pulmonary disease (COPD). CT chest typically featured patchy bibasilar consolidations and subpleural opacities. His COVID19 result came as positive. On examination, the patient was unresponsive, aphasic with deteriorated cognition level. He did not have any gross motor deficiency. There was no nuchal rigidity. The CT scan of the head showed presence of an area of encephalomalacia in the left temporal region, which was most likely due to his previous cerebral infarction. The EEG showed bilateral slowing and focal slowing in the left temporal region with sharply countered waves. Cerebrospinal fluid (CSF) analysis by lumbar puncture was unremarkable for central nervous system infection. Later on, the patient developed respiratory failure and required intubation and was transferred to the ICU.

One single-institute cross-sectional study was performed on 59 COVID-19 positive and 203 COVID19 negative patients who presented with flu like symptoms. Chemosensory Dysfunction was compared in both the groups. ${ }^{20}$ Smell and taste loss were respectively reported in $68 \%$ and $71 \%$ of COVID19 -positive subjects, compared to $16 \%$ and $17 \%$ of COVID-19-negative patients $(p<0.001)$. The result was statistically significant. Smell and taste impairment were independently and strongly associated with Covid-19-positivity. It was concluded that otherwise asymptomatic cases with influenza-like symptoms, chemosensory dysfunction was strongly associated with Covid-19 infection and should be considered when screening symptoms.

Rong Yin et al published their case report on a 64year old male from Wuhan city who had complaint of raised temperature and mild cough. ${ }^{21}$ Then on day 11 of symptom he started becoming lethargic and impaired conscious level. CT scan of brain was unremarkable but CT scan of chest showed peripheral opacities. His oxygen saturation was $88 \%$ without oxygen and $98 \%$ with 5 -liter oxygen by nasal cannula. Throat swab sent on day 12 reported as COVID-19 positive. His signs of meningeal irritation like neck rigidity, Brudzinski sign and upper motor neuron lesion signs like Babinski and Chaddock were positive. Eventually lumbar puncture was done for CSF analysis and resulted as intracranial pressure of $200 \mathrm{~mm}$ of $\mathrm{H} 2 \mathrm{O}$ with colorless and clear CSF. CSF protein was $275.5 \mathrm{mg} / \mathrm{L}$ and other CSF parameters were normal. The CSF was tested negative for the 2019-nCoV nucleic acid test. He started improving from day 27 of symptoms and gradually his CT chest became normal. His conscious level and neurological findings improved markedly. He was able to maintain $99 \%$ oxygen saturation without any oxygen supplement. His consecutive two throat swab test reported negative on day 27 and day 29.

Autopsy report of COVID19 patients revealed brain edema and cerebral degeneration. ${ }^{22}$ For the first time 
in the pandemic period COVID19 patient presented with viral encephalitis. It was also confirmed by genome sequence that SARS-CoV-2 was present in the CSF of affected patient. It is evident that ACE2 is essentially required for SARS-CoV-2 to infect cells. ${ }^{23}$ The function of ACE2 signaling is to lower blood pressure. Hypertension patients has reduced expression of ACE2 that is why the ability of ACE2 to lower blood pressure is significantly reduced in COVID19 patients. After getting infected by SARS-CoV-2, the function and expression of ACE2 proteins get reduced. As because the expression of ACE2 in hypertensive patients are already low so COVID-19 infection enhances cerebral hemorrhage in these patients.

According to a hypothesis, patients with COVID-19 frequently suffer from coagulopathy and prolonged prothrombin time, both of which are responsible for secondary cerebral hemorrhage. COVID-19 patients may have increased in D-dimers, which can lead to thrombotic vascular events. ${ }^{24}$ Corona Virus (CoV) infection has been proposed as a contributing factor in the pathogenesis of multiple sclerosis (MS). ${ }^{25}$ This hypothesis was supported by findings from studies. Corona-virus like particles have been detected in autopsied brain tissue from a patient with MS. CoV was isolated in autopsied brain tissue from 2 patients with MS. Another study reported intrathecal synthesis of antibodies to human CoV which suggested CNS infection. Human CoV RNA has also been detected from the CSF of patients suffering from MS. ${ }^{26}$

Previous studies documented presence of viruses in the brain of SARS patients especially in the neurons. Experimental studies using transgenic mice further uncovered that either SARS CoV34 or MERS COV, when given intranasally, could enter the brain, perhaps through the olfactory nerves, and from there on quickly spread to some particular parts of brain including thalamus and brainstem. ${ }^{27}$ It is critical that in the mice infected with low virulence of MERS CoV infection particles were recognized distinctly in the cerebrum, however not in the lung, which shows that the CNS infection was significant for the high mortality in infected mice. Among the infected brain regions, the brainstem has been exhibited to be intensely involved by SARS CoV or MERS CoV. ${ }^{27}$ In the brainstem most infected regions included the nucleus of the solitary tract and nucleus ambigus. The nucleus of the solitary tract receives sensory information from the mechanoreceptors and chemoreceptors in the lung and respiratory tracts. These neuroanatomic interconnections highlight that the death of CoV infected animals or patients may occur due to the damage of the cardiorespiratory vital center in the brainstem. ${ }^{28}$

\section{Conclusion:}

COVID-19 is a Corona virus like SARS and MERS viruses. Patients suffering from this disease can present with different symptoms including neurological manifestations. Some patients with $\mathrm{ICH}$ directly seek neurosurgical attention and few patients with respiratory failure or encephalitis may require Neurosurgical ICU support for measuring intracranial pressure (ICP). Few patients may present exclusively with neurological symptoms without having any flue like symptoms. Previous studies showed evidences of encephalitis, multiple sclerosis, cerebral hemorrhage and importantly respiratory failure in Corona affected patients. Enough animal and human studies are required support these findings in case of COVID-19.

\section{Conflict of interest:}

None of the co-authors declared any conflict of interest related to this article.

\section{References:}

1. Abdul Mannan Baig, Areeba Khaleeq, Usman Ali, Hira Syeda. Evidence of the COVID-19 Virus Targeting the CNS: Tissue Distribution, Host"Virus Interaction, and Proposed Neurotropic Mechanisms. ACS Chem Neurosci. 2020 Apr 1;11(7):995-998. doi: 10.1021/ acschemneuro.0c00122.

2. Chaolin Huang, Yeming Wang, Xingwang Li, Lili Ren, Jianping Zhao, Yi Hu et al. Clinical features of patients infected with 2019 novel coronavirus in Wuhan, China. Lancet 2020; 395: 497-506. DOI: 10.1016/S01406736(20)30183-5.

3. Netland, J., Meyerholz, D. K., Moore, S., Cassell, M., and Perlman, S. (2008) Severe acute respiratory syndrome coronavirus infection causes neuronal death in the absence of encephalitis in mice transgenic for human ACE2. J. Virol. 82 (15), 7264"75.

4. Li, Y. C., Bai, W. Z., and Hashikawa, T. (2020) The neuroinvasive potential of SARS-CoV2 may be at least partially responsible for the respiratory failure of COVID19 patients. J. Med. Virol., DOI: 10.1002/jmv.25728.

5. Ali A.Asadi-Pooya, LeilaSimani. Central nervous system manifestations of COVID-19: A systematic review. Journal of the Neurological Sciences. 2020. 413;116832. DOI: 10.1016/j.jns.2020.116832 
6. Giacomelli A, Pezzati L, Conti F, Bernacchia D, Siano M, Oreni L. Self-reported Olfactory and Taste Disorders in Patients With Severe Acute Respiratory Coronavirus 2 Infection: A Crosssectional Study. Clin Infect Dis. 2020.

7. Hopkins C, Surda P, Whitehead E, Kumar B. Early recovery following new onset anosmia during the COVID-19 pandemic - an observational cohort study. J Otolaryngol Head Neck Surg. 2020;49:423-428.

8. Klok F, Kruip M, van der Meer N, Arbuos M, Gommers D, Kant K. Incidence of thrombotic complications in critically ill ICU patients with COVID-19. Thromb Res. 2020;S00493848(20):30120-30121.

9. Lechien J, Chiesa-Estomba C, De Siati D, Horoi M, Le Bon $\mathrm{S}$, Rodriguez A. Olfactory and gustatory dysfunctions as a clinical presentation of mild-to-moderate forms of the coronavirus disease (COVID-19): a multicenter European study. Eur Arch Otorhinolaryngol. 2020; 277:2251-2261.

10. Lodigiani C, Lapichino G, Carenzo L, Cecconi M, Ferrazzi $\mathrm{P}$, Sebastian T. Venous and arterial thromboembolic complications in COVID-19 patients admitted to an academic hospital in Milan, Italy. Thromb Res. 2020;191:914.

11. L. Mao, M. Wang, S. Chen, et al., Neurological Manifestations of Hospitalized Patients with COVID-19 in Wuhan, China: a Retrospective Case Series Study, https:// www.medrxiv.org/content/10.1101/2020.02.22. 20026500v1/.

12. Y. Li, M. Wang, Y. Zhou, et al., Acute Cerebrovascular Disease Following COVID-19: A Single Center, Retrospective, Observational Study, https:// papers.ssrn.com/sol3/ papers.cfm.abstract_id=3550025/ , (2020).

13. C. Huang, Y. Wang, X. Li, et al., Clinical features of patients infected with 2019 novel coronavirus in Wuhan, China, Lancet 395 (2020) 497-506.

14. X. Yang, Y. Yu, J. Xu, et al., Clinical course and outcomes of critically ill patients with SARS-CoV-2 pneumonia in Wuhan, China: a single-centered, retrospective, observational study, Lancet Respir. Med. (2020 Feb 24), https://doi.org/10.1016/ S2213-2600(20)30079-5 pii: S2213-2600(20)30079-5. In press.

15. D. Wang, B. Hu, C. Hu, et al., Clinical characteristics of 138 hospitalized patients with 2019 novel coronavirusinfected pneumonia in Wuhan, China, Jama (2020 Feb 7), https://doi.org/10.1001/jama.2020.1585 In press.

16. N. Chen, M. Zhou, X. Dong, et al., Epidemiological and clinical characteristics of 99 cases of 2019 novel coronavirus pneumonia in Wuhan, China: a descriptive study, Lancet 395 (2020) 507-513.

17. Sijia Tian, Nan Hu, Jing Lou, Shengmei Niu, Luxi Zhang, Jinjun Zhang et al. Characteristics of COVID-19 Infection In Beijing. Journal of Infection. 2020 ; 80(4) :401-406.

18. Sandeep Sohal and Mossammat Mansur. COVID-19 Presenting with Seizures. ID Cases. IDCases. 2020; 20 : e00782. doi: 10.1016/j.idcr.2020.e00782.

19. Asia Filatov, Pamraj Sharma, Fawzi Hindi and Patricio S Espinosa. Neurological Complications of Coronavirus Disease (COVID-19): Encephalopathy. Cureus. 2020; 12(3): e7352. doi: 10.7759/cureus.7352.

20. Carol H. Yan, Farhoud Faraji, Divya P. Prajapati, Christine E. Boone, Adam S DeConde. Association of Chemosensory Dysfunction and Covid-19 in Patients Presenting with Influenza-like Symptoms. Wiley Online Library. March 2020. Doi: $10.1002 / a l r .22579$.

21. Rong Yin, Wei Feng, Tonghui Wang, Gang Chen, Tao Wu, Dongrui Chen et al. Concomitant neurological symptoms observed in a patient diagnosed with coronavirus disease 2019. Journal of Medical Virology. 2020 March. Doi: 10.1002/jmv.25888

22. Xiang P., Xu X.M., Gao L.L., Wang H.Z., Xiong H.F., Li R.H. First case of 2019 novel coronavirus disease with Encephalitis. ChinaXiv. 2020;T202003:00015.

23. Hai-Yang Wang, Xue-Lin Li, Zhong-Rui Yan. Potential neurological symptoms of COVID-19, Therapeutic Advances in Neurological Disorders. March 2020. doi:10.1177/1756286420917830

24. Xu X, Chen P, Wang J, et al. Evolution of the novel coronavirus from the ongoing Wuhan outbreak and modeling of its spike protein for risk of human transmission. Sci China Life Sci. Epub ahead of print 21 January 2020. DOI: 10.1007/ s11427-020-1637-5.

25. Talbot P.J. Virus-induced autoimmunity in multiple sclerosis: The coronavirus paradigm. Adv Clin Neurosci. 1997;7:215233.

26. Bonavia A., Arbour N., Yong V.W., Talbot P.J. Infection of primary cultures of human neural cells by human coronaviruses 229E and OC43. J Virol. 1997;71:800-806.

27. Li K, Wohlford Lenane C, Perlman S, et al. Middle East respiratory syndrome coronavirus causes multiple organ damage and lethal disease in mice transgenic for human dipeptidyl peptidase 4. J Infect Dis. 2016;213:712 722.

28. Matsuda $\mathrm{K}$, Park $\mathrm{CH}$, Sunden $\mathrm{Y}$, et al. The vagus nerve is one route of transneural invasion for intranasally inoculated influenza a virus in mice. Vet Pathol. 2004;41:101 107. 